\section{ARS Tech}

Authors retain copyright

\section{Applied Research and Smart Technology}

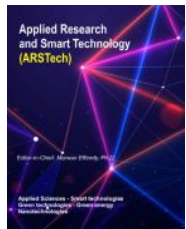

ISSN 2722-9645

Research Article

\title{
An experimental study of the dynamic properties of smart composite magnetorheological materials
}

\author{
Aguib Salah ${ }^{1}$, , Rouabah Salah ${ }^{2}$, Hadji Mohamed ${ }^{2}$, Kobzili Lallia ${ }^{1}$, Chikh Noureddine ${ }^{1}$ and Toufik
} Djedid $^{1}$

${ }^{1}$ Dynamic Motors and Vibroacoustic Laboratory, University of Boumerdes. 35000, Algeria.

${ }^{2}$ Laboratory of Studies and Research in Industrial Technology, University of Saad Dahleb Blida 1, Algeria.

*Corresponding author: s.aguib@univ-boumerdes.dz

Permalink (DOI): https://doi.org/10.23917/arstech.v2i1.218

\begin{tabular}{|c|c|}
\hline ARTICLE INFO & ABSTRACT \\
\hline Article history: & A magnetorheological is a new generation of smart materials in active mechanical \\
\hline Received 17 December 2020 & vibration and shock control applications. This article is based on a comparative \\
\hline Revised 13 March 2021 & experimental study of the dynamic properties of magnetorheological elastomers \\
\hline Accented 18 March 2021 & (MRE) and magnetorheological fluids (MRF), whose damping and stiffness are \\
\hline Accepted 18 March 2021 & controlled simultaneously by applying magnetic field intensity. The dynamic \\
\hline Avalladie onme $2 \angle$ June $20 \angle 1$ & visco-analyser and rheometer were used to test the behaviour of MRE and MRF. \\
\hline & $\begin{array}{l}\text { The response of both materials depends on the amplitude due to shear strain. } \\
\text { The experimental results clearly show the difference between the dynamic }\end{array}$ \\
\hline Keywords: & properties of MRE and MRF. More specifically, MFRs have high damping with \\
\hline Dynamic properties & low stiffness; on the other hand, the MRE has average damping with outstanding \\
\hline Loss factor & rigidity. These results help designers achieve more efficient and reliable structures \\
\hline Magnetic field intensity & in various engineering fields, such as buildings and mechanical applications. \\
\hline
\end{tabular}

Magnetorheological materials

Rigidity

\section{INTRODUCTION}

Innovative materials such as magnetorheological fluids (MRF) and magnetorheological elastomers (MRE) have become preferred materials in many applications due to the ability to change properties in a controlled manner under the application of stimulation such as variable current, magnetisation, heat, force, stress, and strain. In addition, the magnetic field controls the physical change of the mixture to the semi-solid state, making these materials a reliable element in active vibration and mechanical shock control applications.

In recent years, worm-like micelles (WLMs) have been used by Ghatee et al. [1] to improve the properties of water-based MRFs. The study used Cetyl trimethyl 
ammonium bromide (CTAB) as a dispersant. Lampaert et al. [2] evaluated a hydrostatic bearing in which the pressure profile of a conventional hydrostatic bearing is recreated with only the use of a magnetic field and a magnetorheological fluid. Jian-Feng et al. [3] studied a magnetorheological liquid to recycle the oil slick efficiently. A core-shell material of magnetic nanoparticles (MPs) of $\mathrm{Fe} 3 \mathrm{O} 4$ coated with oleic acid (OA) was uniformly dispersed in a base fluid.

Vishnyakov et al. [4] have developed a new independent description of flows in plastic and viscous regions that could be used to study magnetohydrodynamic flows of Bingham fluid. The design of an MR damper, consisting of a piston and cylinder arrangement, was presented by Mangal et al. [5]. Xufeng et al. [6] used a DC Arc discharge method to prepare iron nanoparticles. The morphology, magnetic properties, crystal structures and surface chemical composition were examined. Sahoo et al. [7] have shown that the values of dynamic yield stress corresponding to different applied magnetic field intensities increase with increasing magnetic field intensity. Pant et al. [8] studied the effect of ferrofluid incorporated $(5-20 \% \mathrm{v} / \mathrm{v})$ flake-shaped iron particles in silicon oil-based magnetorheological fluid (MR fluid).

In the work of Zhong-jin et al. [9], numerical analysis on magnetorheological elastomers bulging for sheet metal was carried out. The obtained results indicated that the magnetorheological elastomer's shear modulus increases with the magnetic intensities. Tong et al. [10] prepared Flower-like Cobalt Particle-Based MRE (FCPMRE), Spherical Cobalt Particle-Based MRE (SCPMRE), and Flower-like MRE Coupling Agent modified cobalt (M-SCP-MRE) for comparison. Recent advances in $\mathrm{X}$-ray microtomography allow for the mutual evaluation of large particles so that the PCF can be applied to tomographically captured three-dimensional particle structures [11].

An external stimulus activates MREs, an appropriate candidate for $4 \mathrm{D}$ printing where a shape-shifting of a 3D printed structure under external stimuli over time was needed [12][13]. Michal et al. [14] have developed a composite material based on a geopolymer matrix and a reinforcement of basalt fibres. Bodnaruk et al. [15] have shown that the constant magnetic anisotropy resulted from particle displacements and a characteristic of the energy of internal deformations in the polymer matrix.

Agirre-Olabide et al. [16] have developed a new magneto-viscoelastic model for anisotropic magnetorheological elastomers (MREs), combining dynamic behaviour and magnetic permeability components. Zuzana et al. [17] have studied the effects of the reinforcement phase in particulate ceramic composites on their properties. Nedjar and al. [18] determined the mechanical characteristics of a magnetorheological elastomer loaded with 20,30 and 40\% iron particles.

The effect of temperature on the dynamic mechanical properties of MR elastomers has been examined by Wan et al. [19]. The impact of the nature of the particles on the mechanical properties and the magnetic effect of elastomers MRE have been studied by Kumar et al. [20]. Wang et al. [21] have developed nanocomposites of cobalt ferrite (CoFe2O4) nanoparticles grown on multi-walled carbon nanotubes (MWCNT) by the ultrasound-assisted coprecipitation technique. Kumar et al. [22] used two magnetisable particles (carbonyl iron (CI) and electrolytic iron (EI)). These two particles were collected and characterised using an Anton Paar MCR 702 rheometer setup in on and off states. Niu et al. [23] discussed a study of the dynamic analysis and vibration control of the boring bar system with magnetorheological fluid control. In this article, Zhang et al. [24] performed a numerical study on domed magnetorheological elastomers for sheet metal. The speed and stress distribution of the sheet under different magnetic intensities were compared and analysed. Janbaz et al. [25] experimentally and numerically studied the magneto-hyper-viscoelastic responses of isotropic magnetorheological elastomers (MREs) subjected to uniaxial compressive loads induced by magnetic fields.

This article, a comparative study between magnetorheological elastomers and magnetorheological fluids, aims to help engineers exploit these recent materials in different fields of application by identifying their dynamic properties as a function of the shear strain amplitude experimentally.

\section{ANALYTICAL MODELING}

A micromechanical analytical model has been developed on an elementary cell that can generate a representative chain by replicating along the chain's axis to describe the behaviour of the magnetorheological elastomer composite. This cell is a cylinder, a real agglomerate of the composite; it consists of two hemispheres coated in a large elastomer cylinder. In reality, the magnetorheological composite has a very complex microstructure; one can think of aggregates disturbing the arrangement of chains, misalignment, and length. There are columnar aggregates formed of several particle thicknesses. An electron microscope could observe the finest area, and the area separating the particles increases.

The approach adopted isolates the essential physical parameters for this type of system: the anisotropic structure, the gap separating two particles within the same chain, and the volume fraction of the charges. Thus, the overall design is made of particles ideally spaced by the 
same gap, making the ratio of the gap over the radius of the particles the essential parameter of this model. The volume fraction $\phi$ fixes the inter-chain distance, represented here by the quantity $\mathrm{L}$. $\mathrm{L}$ is the thickness of the elastomer ring surrounding the particles and is calculated as a function of the ratio $\mathrm{g} / \mathrm{a}$ and of the volume fraction $\phi$ of inclusions, given as follows:

$\phi=\frac{V_{\text {Particles }}}{V_{\text {Matrice }}+V_{\text {Particles }}}$

In the study, experiments were conducted to characterise the dynamic properties of the MRE over the strain amplitude and magnetic flux density. The loss angle was determined by:

$\delta=\frac{G^{\prime \prime}}{G^{\prime}}$

Where:

$G^{\prime}=G^{*} \cos (\delta)$

$G^{\prime \prime}=G^{*} \sin (\delta)$

The complex modulus $(\mathrm{G})$ is the ratio of the shear stress $\tau(\mathrm{t})$ and shear strain $\gamma(\mathrm{t})$, given as follows:

$\phi=\frac{\tau_{a}}{\gamma_{a}}$

Therefore, the loss modulus could be estimated from the energy dissipation corresponding to the hysteresis loop area [26]. Thus, the energy dissipation $\mathrm{D}$ per loading cycle is expressed as:

$D=\int_{0}^{T} \tau(t) \dot{\gamma}(t) d t=\pi \gamma_{a}^{2} G^{*} \sin (\delta)=\pi \gamma_{a}^{2} G^{\prime \prime}$

\section{MATERIALS AND METHOD}

\subsection{Magnetorheological fluid}

MRF is a smart material that reacts almost instantly to an applied magnetic field by changing its rheological properties. MRF is one of the categories of fluids sensitive to applying a magnetic field. It consists of ferromagnetic particles (spherical or elliptical in shape) of the order of a micrometre from 0.1 to $10 \mu \mathrm{m}$ bathed in a carrier liquid, also called a solvent. A variety of carrier fluids, such as mineral oil, could be found, most organic. It is large enough particles to accommodate multiple magnetisable domains (Weiss domains).

Without a magnetic field, the spin orientations relating to each part were randomly arranged. Thus, the resulting magnetisation at the particle level was zero. There was, therefore, no magnetic interaction force between two or more ferromagnetic suspensions. Consequently, the metal particles were homogeneously dispersed within the carrier liquid: the resulting viscosity is very low but constant during shearing.

On the other hand, when this fluid was subjected to an external magnetic field of the order of a Tesla fraction, the spin moments tended to orient themselves in the same direction as the field (by minimisation of the interaction energy). There then appeared a magnetic interaction force was joining between particles. The apparent viscosity $\eta$ of the fluid increased very sharply, so much so that it behaved like a viscoelastic solid. The ferromagnetic particles then lined up in the same direction as the magnetic field lines and formed actual chain structures.

\subsection{Magnetic device for MRF}

Figure 1 shows the Couette-plan rheometer. In experiments, a sample of magnetic fluid was placed in the air gap to occupy all the available space. Note that the field was saturated because an iron core drives it. As air bubbles in the fluid were a source of error, it must be careful to burst them. The rheometer imposed increased stress in the air gap, then the acquisition software attached to the rheometer calculated the speed at which the magnetic fluid shifts.

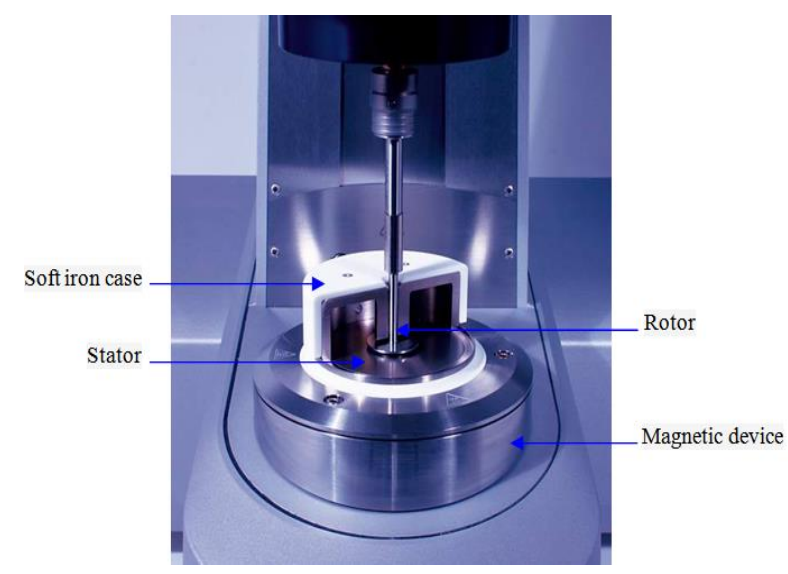

Figure 1. Couette-plan rheometer.

\subsection{Magnetorheological elastomer}

A Dynamic Mechanical Analyser (DMA) machine was used to perform dynamic tests on small samples by varying the frequency. The objective was to investigate the role of microstructure on the macroscopic response of the elastomer composite when having the magnetomechanical loading conditions. The dynamic mechanical analysis (MetraviB DMA +450) was applied to determine the rheological properties of MRE. The Metravib DMA +450 consists of a very rigid frame and a movable punch, as in Figure 2. 


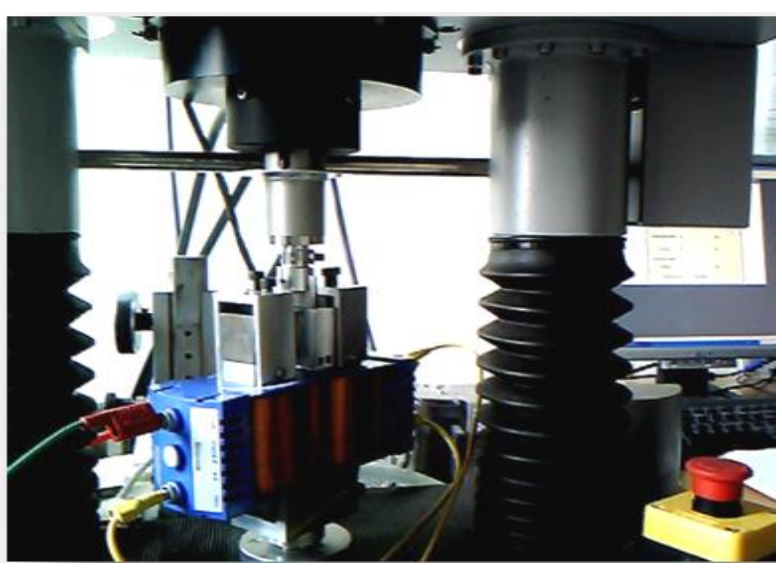

Figure 2. Shear test by Viscoanalyser Metra viB DMA $450^{+}$with an MRE specimen in the electromagnet system.

\section{RESULTS AND DISCUSSION}

Experiments have been conducted on the dynamic properties of magnetorheological elastomers (MRE) and magnetorheological fluids (MRF). The key results and findings are discussed as follows.

Experimental data of MR fluid and MR elastomer with a volume fraction of $30 \%$ under an applied magnetic field of $400 \mathrm{mT}$ are given in Table 1 and Table 2.

Table 1. Experimental data of MR elastomer with volume fraction of $30 \%$ under applied magnetic field $400 \mathrm{~m}$ T.

\begin{tabular}{cccc}
\hline Shear strain & $\mathrm{G}^{\prime}(\mathrm{Pa})$ & $\mathrm{G}^{\prime \prime}(\mathrm{Pa})$ & $\eta$ \\
\hline 0.00483404 & 2917610.88 & 736339.776 & 0.25237765 \\
0.00726212 & 2526391.87 & 653690.304 & 0.25874462 \\
0.00969288 & 2294115.07 & 594897.638 & 0.25931465 \\
0.01931790 & 1816645.63 & 468517.632 & 0.25990260 \\
0.03391860 & 1511861.57 & 384718.234 & 0.26066570 \\
0.04878650 & 1346509.06 & 344926.138 & 0.26056325 \\
\hline
\end{tabular}

Table 2. Experimental data of MR fluid with volume fraction of $30 \%$ under applied magnetic field $400 \mathrm{mT}$.

\begin{tabular}{cccc}
\hline Shear strain & $\mathrm{G}^{\prime}(\mathrm{Pa})$ & $\mathrm{G}^{\prime \prime}(\mathrm{Pa})$ & $\eta$ \\
\hline 0.0104406 & 402574.2336 & 529861.9200 & 1.316184385 \\
0.0104407 & 346239.6800 & 347239.6800 & 1.002888173 \\
0.0104408 & 291574.2339 & 254424.7872 & 0.872590090 \\
0.0104409 & 251574.2338 & 202388.2368 & 0.804487143 \\
0.0104410 & 221574.2337 & 185682.1542 & 0.838013297 \\
0.0104420 & 216574.2337 & 163624.6523 & 0.755513015 \\
\hline
\end{tabular}

Figure 3 shows the optical micrograph of magnetic response (MR) fluids with a magnification factor of 500x. The magnetic particles are dispersed in a carrier fluid.

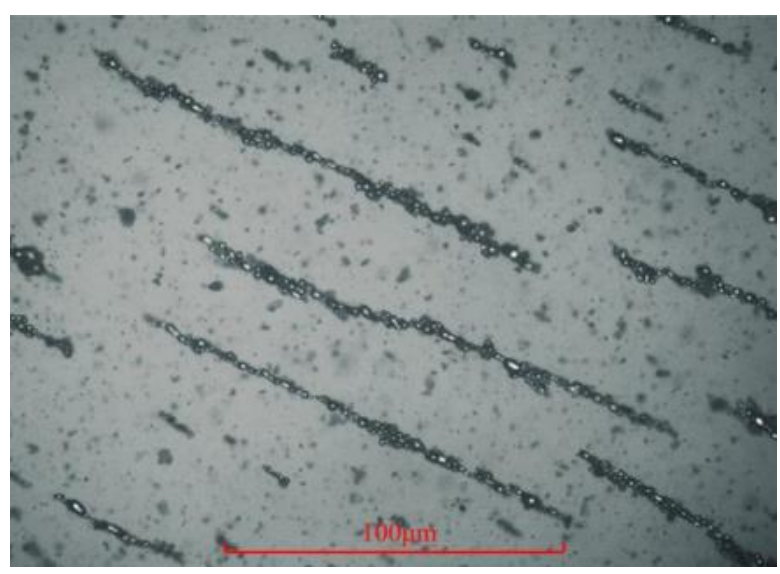

Figure 3. Section of the structured composite magnetorheological fluid observed by optical microscope.

A microscope could observe the finest area, and the area separating the particles increases Figure 4.

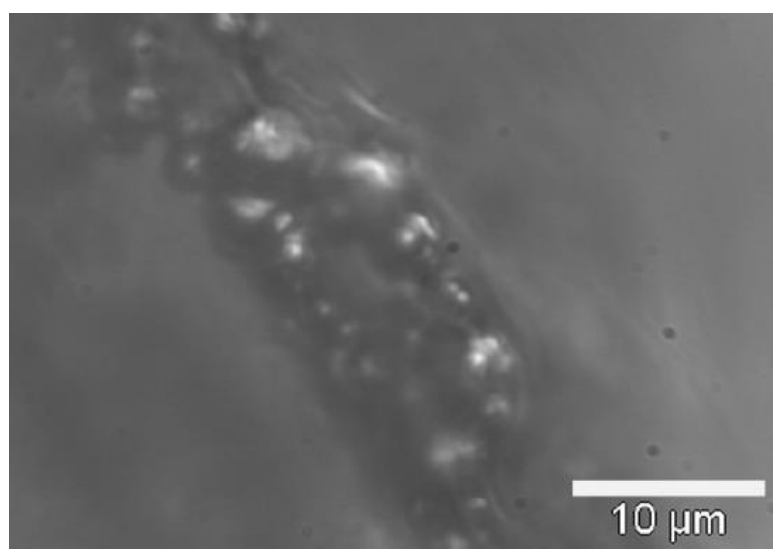

Figure 4. Section of the structured composite magnetorheological elastomer observed by optical microscope.

The results of dynamic tests under a magnetic field with an intensity of $400 \mathrm{mT}$ are presented in Figures 5, 6 and 7.

Figure 5 indicates the variation of the storage modulus according to the shear strain energy. The storage modulus decreases due to the increase in the shear strain energy. The increase in rigidity of the MRE causes a decrease in storage modulus. This matches the increased magnetic force between ferromagnetic particles and the reduction in energy stored by weak sliding between iron particles [8].

On the other hand, this modulus decreases with the increase of the strain energy in shear. The material's yield stress decreases due to the rise of the energy losses in the 
suspensions. The inter-particles solid surface friction also causes it by smoothing the surface of particles in a flake shape.

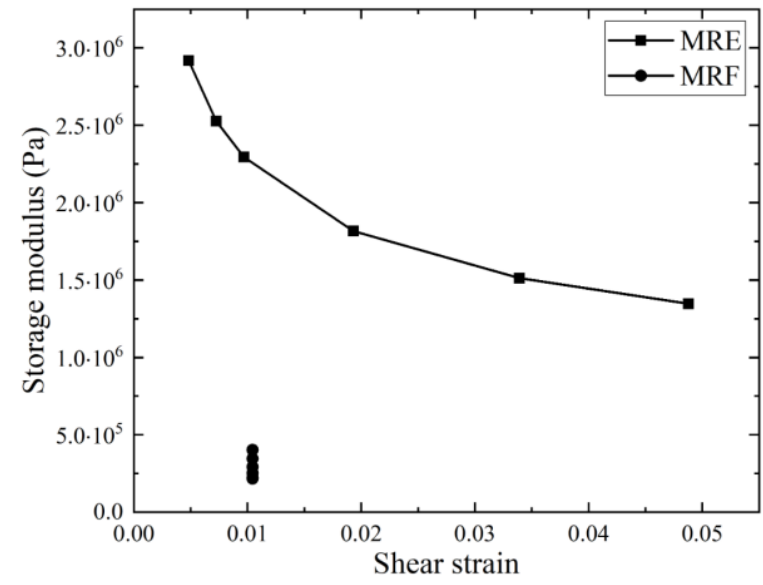

Figure 5. Variation of storage modulus as a function of shear strain energy.

Figure 6 give loss modulus as a function of shear strain energy. The shear strain energy increase leads to a significant decrease in the loss modulus for the magnetorheological elastomer, and this decrease in the loss modulus is more pronounced for higher shear strain energy values. A significant reduction in the loss modulus is also observed in a magnetorheological fluid. This decrease is mainly due to the increase in the sliding effect between the ferromagnetic particles and the decline in rigidity according to the increase in viscosity.

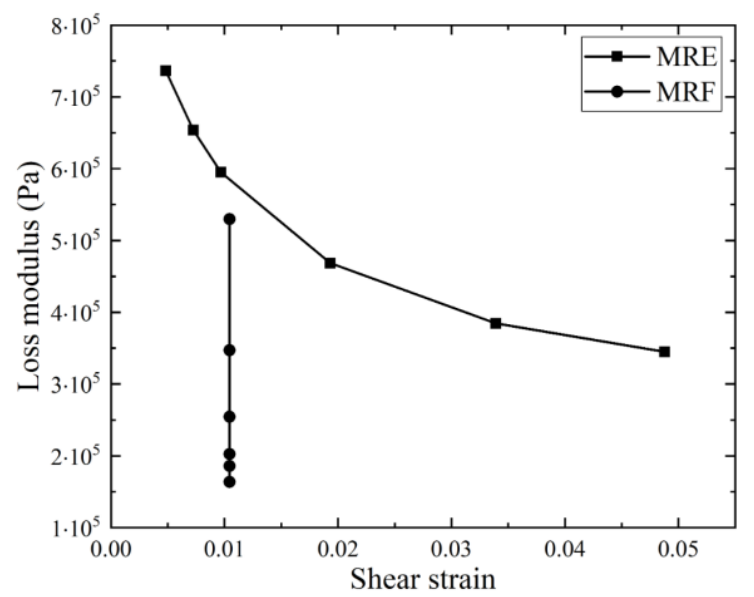

Figure 6. Variation of loss modulus as a function of shear strain energy.

Figure 7 illustrates the evolution of the loss factor according to the shear strain energy for the two materials MRE and FMR. Note that the loss factor increases with the increase in the shear strain energy; this increase is due to the increase in rigidity, i.e., the decrease in the ratio loss modulus/storage modulus. On the other hand, the loss factor decreases with the increase in the shear strain energy. This decrease is due to reducing viscosity at the saturation point and decreasing the ratio loss modulus/storage modulus.

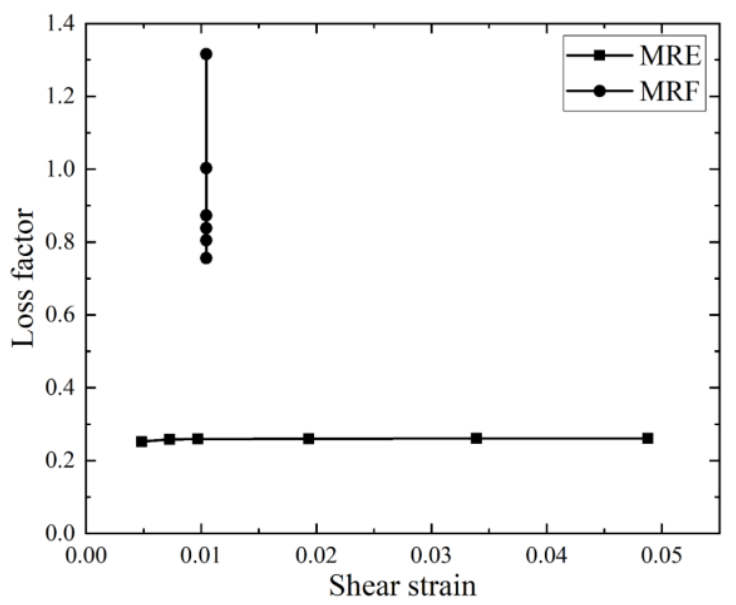

Figure 7. Variation of loss factor as a function of shear strain energy.

This work is devoted to a comparative study of magnetorheological materials (MRE and MRF) from a mechanical perspective. The MRE is made up of iron particles subjected to a magnetic field. Under the magnetic field effect, the particles polarise and align in the direction of the area. The spatial distribution of particles by the magnetic field is responsible for changes in the material's mechanical properties. Due to their fibrillar structure, MRE materials are more interesting than MRF materials: they are conductors even at a low particle charge ratio.

On the other hand, many application fields use MRFs to dissimulate mechanical energy. Their ability to rapidly change their properties, depending on a magnetic field, makes these fluids an excellent way to control the static and dynamic behaviour of various mechanical devices such as shock absorbers, brakes and clutches. However, these mechanical energy dissipators are required to operate in environments subject to external disturbances, particularly vibrations.

\section{CONCLUSION}

The magnetorheological fluid (MRF) and the magnetorheological elastomer (MRE) are two intelligent magnetorheological materials. It has been developed from certain products which are commercialised and used in practice. From this work, it can be concluded that magnetorheological fluids (FMRs) have an excellent variable damping property, and MRE changes their stiffness in milliseconds in the presence of a magnetic field. MRF and MRE materials are of great interest in realising damping variability or stiffness variability for vibration control and mechanical shock remediation in a wide range of civil (buildings) and mechanical structures (automotive). 


\section{CONFLICTS OF INTEREST}

The authors declare that no competing financial interests or personal relationships could have appeared to impact the work reported in this paper.

\section{ACKNOWLEDGEMENT}

The authors would like to thank the University of Boumerdes Algeria and the University of Saad Dahleb Blida 1 Algeria for supporting the research facilities.

\section{REFERENCES}

[1] M. Ghatee, A.H. Dorostia, and M. Norouzi, "Preparation and characterisation of water-based magnetorheological fluid using worm-like surfactant micelles", Journal of Magnetism and Magnetic Materials, $\quad$ Vol. 498, 2020, https://doi.org/10.1016/j.jmmm.2019.166193.

[2] S.G.E. Lampaert, and R.A.J. Van Ostayen, "Experimental results on a hydrostatic bearing lubricated with a magnetorheological fluid", Current Applied Physics, Vol. 19, pp. 1441-1448, 2019, https://doi.org/10.1016/j.cap.2019.09.004.

[3] Z. Jian-Feng, T. Feng, S. Chun-Lei, W. Hong-Bo, and H. Liang, "Effective recovery of oil slick using the prepared high hydrophobic and oleophilic Fe3O4 magnetorheological fluid", Colloids and Surfaces A: Physicochemical and Engineering Aspects, vol. 591, 2020, https://doi.org/10.1016/j.colsurfa.2020.124531.

[4] V.I. Vishnyakov, S.M. Vishnyakova, P.V. Druzhinin, and L.D. Pokrovskii, "Unsteady flow of an electrically conducting bingham fluid in a plane magnetohydrodynamic channel", Journal of Applied Mechanics and Technical Physics, Vol. 60, pp. 432437,

2019 , https://doi.org/10.1134/S0021894419030040.

[5] SK. Mangal, and K. Ashwani, "Experimental and numerical studies of magnetorheological (MR) damper", Chinese Journal of Engineering, 2014, https://doi.org/10.1155/2014/915694.

[6] D. Xufeng, Z. Wanning, H. Hao, and Q. Min, "Iron nanoparticles-based magnetorheological fluids: A balance between MR effect and sedimentation stability", Journal of Magnetism and Magnetic Materials, Vol. 491, 2019, https://doi.org/10.1016/j.jmmm.2019.165556.
[7] B. Sahoo, AV Anupama, and V. Kumaran, "Application of Ni-Zn ferrite powders with polydisperse spherical particles in magnetorheological fluids", Powder Technology, Vol. 338, pp. 190-196, 2018, https://doi.org/10.1016/j.powtec.2018.07.008.

[8] R.P. Pant, J. Noor, P. Saurabh, and J. Komal, "Enchancment in viscoelastic properties of flakeshaped iron based magnetorheological fluid using ferrofluid", Colloids and Surfaces A: Physicochemical and Engineering Aspects, Vol. 529, pp. 88-94, 2017, https://doi.org/10.1016/j.colsurfa.2017.05.057.

[9] W. Zhong-jin, Z. Rui, Y. Chao, and L, Xiao, "The numerical analysis of the magnetorheological elastomer bulging for sheet metal", Procedia Manufacturing, Vol. 50, pp. 110-113, 2020, https://doi.org/10.1016/j.promfg.2020.08.020.

[10] Y. Tong, D. Xufeng and Q. Min, "Payne effect and damping properties of flower-like cobalt particlesbased magnetorheological elastomers", Composites Communications, Vol. 15, pp. 120-128, 2019, https://doi.org/10.1016/j.coco.2019.07.007.

[11] M. Schümann, and S. Odenbach, "In-situ observation of the particle microstructure of magnetorheological elastomers in presence of mechanical strain and magnetic fields", Journal of Magnetism and Magnetic Materials, Vol. 441, pp. 88-923, 2017 , https://doi.org/10.1016/j.jmmm.2017.05.024.

[12] T. Van Manen, S. Janbaz, and A.A. Zadpoor, "Programming the shape-shifting of flat soft matter", Materials Today, Vol. 21, pp. 144-163, 2018, https://doi.org/10.1016/j.mattod.2017.08.026.

[13] T. Van Manen, S. Janbaz, and A.A. Zadpoor, "Programming 2D/3D shape-shifting with hobbyist 3D printers", Materials Horizon, Vol. 4, pp. 1064 1069 , 2017 , https://doi.org/10.1039/C7MH00269F.

[14] M. S. Michal, L. Petr, E. Petr, L. C. Hiep, K. Vladimír, V.S. Le, V. Lukášs, B. Elif, and B. Totka, "Evaluation of mechanical properties of composite geopolymer blocks reinforced with basalt fibres", Manufacturing Technology, Vol. 18(5), pp. 861-865, 2018, https://doi.org/10.21062/ujep/191.2018/a/12132489/MT/18/5/861.

[15] A.V. Bodnaruk, A. Brunhuber, V.M. Kalita, M.K. Mykola, K. Peter, A.S. Andrei, F.L. Albert, M.R. Sergey, and S. Mikhail, "Magnetic anisotropy in magnetoactive elastomers, enabled by matrix elasticity", Polymer, Vol. 16 (2), pp. 63-72, 2019, https://doi.org/10.1016/j.polymer.2018.12.027. 
[16] I. Agirre-Olabide, P. Kuzhir, and M.J. Elejabarrieta, "Linear magneto-viscoelastic model based on magnetic permeability components for anisotropic magnetorheological elastomers", Journal of Magnetism and Magnetic Materials, Vol. 446, pp. 155-161, 2018 https://doi.org/10.1016/j.jmmm.2017.09.017.

[17] G. Zuzana, Š. Pavol, and B. Alena, "Microstructure and selected properties of $\mathrm{Si} 3 \mathrm{~N} 4+\mathrm{SiC}$ composite", Manufacturing Technology, Vol. 20(3), pp. 293-299, 2020, https://doi.org/10.21062/mft.2020.056.

[18] A. Nedjar, S. Aguib, T. Djedid, A. Nour, A. Settet and M. Tourab, "Analysis of the dynamic behavior of magnetorheological elastomer composite: elaboration and identification of rheological properties", Silicon, Vol. 11, pp. 1287-1293, 2019, https://doi.org/10.1007/s12633-018-9921-1.

[19] V. Kumar and D-J. Lee, "Mechanical properties and magnetic effect of new magnetorheological elastomers filled with multi-wall carbon nanotubes and iron particles", Journal of Magnetism and Magnetic Materials, Vol. 482, pp. 329-335, 2019, https://doi.org/j.jmmm.2019.03.075

[20] Y. Wan, Y. Xiong, and S. Zhang, "Temperature dependent dynamic mechanical properties of magnetorheological elastomers: Experiment and modeling", Composite Structures, Vol. 202, pp. 768773 , 2018, https://doi.org/j.compstruct.2018.04.010.

[21] F. Wang, Y. Ma, H. Zhang, J. Gu, J. Yin, X. Jia, H. Zhang, Y. Wang, X. Fu, R. Yu, Z. Wang, S. Han, and G. Wang, "Rheological properties and sedimentation stability of magnetorheological fluid based on multi-walled carbon nanotubes/cobalt ferrite nanocomposites", Journal of Molecular Liquids, Vol. 324, 2021, https://doi.org/10.1016/j.molliq.2020.115103.
[22] J. S. Kumar, D. G. Alex, and P. P. Sam, "Synthesis of magnetorheological fluid compositions for valve mode operation", Materials Today: Proceedings, Vol. 22, pp. 1870-1877, 2020, https://doi.org/10.1016/j.matpr.2020.03.086.

[23] J. Niu, J. Hou, Y. Shen, and S. Yang, "Dynamic analysis and vibration control of nonlinear boring bar with fractional-order model of magnetorheological fluid", International Journal of Non-Linear Mechanics, Vol. 121, 2020, https://doi.org/10.1016/j.ijnonlinmec.2020.10345 2

[24] R. Zhang, C. Yin, X. Luo, and Z. Wang, "The numerical analysis of the magnetorheological elastomer bulging for sheet metal", Procedia Manufacturing, Vol. 50, pp. 110-113, 2020, https://doi.org/10.1016/j.promfg.2020.08.020.

[25] M. Janbaz, and H.S. Googarchin, "Experimental and numerical analysis on magneto-hyperviscoelastic constitutive responses of magnetorheological elastomers: A characterisation procedure", Mechanics of Materials, Vol. 154, 2021, https://doi.org/10.1016/j.mechmat.2020.103712.

[26] T.H. Nam, I. Petríkova, and B. Marvalova, "Experimental characterisation and viscoelastic modeling of isotropic and anisotropic magnetorheological elastomers", Polymer Testing, Vol. 81, pp. 1062-1072, 2020, https://doi.org/10.1016/j.polymertesting.2019.106 $\underline{272}$. 